\title{
Obesity has limited behavioural overlap with addiction and psychiatric phenotypes
}

4

$5 \quad$ Uku Vainik $^{1,2^{*}}$, Bratislav Misic ${ }^{1}$, Yashar Zeighami ${ }^{1}$, Andréanne Michaud ${ }^{1}$, Rene Mõttus ${ }^{3,2}$, Alain $6 \quad$ Dagher $^{1}$

7

1. Montreal Neurological Institute, McGill University, Canada

9

* Corresponding author: uku.vainik@gmail.com

2. Institute of Psychology, University of Tartu, Estonia

3. Department of Psychology, University of Edinburgh, UK (1)

Final version out now at Nature Human Behavior: https://doi.org/10.1038/s41562-019-0752-x 
Obesity is a widespread health condition ${ }^{1}$, likely driven by increased availability of inexpensive high-calorie food ${ }^{2}$. People vary in their behavioural response to food plenty. Such variation is likely driven by behavioural styles ${ }^{3,4}$, as behaviour accounts for entire food intake ${ }^{5}$. A prominent hypothesis is that people with obesity respond to rewards similarly to people with addictions such as alcohol abuse or smoking ${ }^{6,7}$. For instance, perceived overeating or "Uncontrolled Eating" (UE) is the most common obesity-associated personality trait ${ }^{8}$ and resembles the perceived loss of control seen in drug addiction. Likewise, both obesity and addictive behaviours have similar correlations with broad personality domains ${ }^{3}$. Here, we seek to empirically test whether obesity and UE overlap behaviourally with addiction and psychiatric disorders, collectively referred to as phenotypes. We test for behavioural similarity by linking the personality profiles of each phenotype. NEO Personality Inventory (NEO PI-R/3) profiles of 28 phenotypes were extracted from 22 studies, encompassing summary statistics from 18,611 unique participants. Obesity had moderate and UE high behavioural similarity with addictions. UE also overlapped behaviourally with most psychiatric phenotypes, whereas obesity was behaviourally similar with mood disorders and certain personality disorders. Facet-based phenotype profiles provided more information than domain-based profiles.

Vulnerability to drug use and to overeating have been suggested to have a shared behavioural basis $^{3,6,7}$. For instance, drug use and obesity are associated with similar personality questionnaires and cognitive tests ${ }^{3}$. However, the similarities are observational and have not been quantified. Quantifying the behavioural overlap between obesity and addictions has nosological and therapeutic implications, and may reveal underlying behavioural mechanisms and risk factors. 
Addictive features may also characterise a commonly identified eating-related phenotype, Uncontrolled Eating (UE). UE is conceptualised as high food reward sensitivity combined with poor self-control ${ }^{8,}$, and this trait explains most of the variance in the common eating-related questionnaires measuring emotional eating, food addiction, or binge eating ${ }^{8,9}$. Despite these questionnaires’ slightly differing definitions and item content ( ${ }^{\text {reviewed in } 10}$ ), UE-related questionnaires demonstrate similar correlations with other variables, such as obesity ${ }^{8}$ and personality traits ${ }^{10}$. UE may capture aspects of behaviour that resemble addictions, as one of the UE questionnaires, the Yale Food Addiction Questionnaire, is based on the Diagnostic and Statistical Manual of Mental Disorders’ criteria for drug abuse. However, the behavioural similarities between addiction, obesity, and UE have not yet been systematically investigated.

Addictive features likely explain only part of the behavioural repertoire of obesity, as this condition can develop from multiple behavioural paths ${ }^{11}$. Therefore, we also assessed potential behavioural similarities of obesity and UE with other psychiatric conditions. Obesity and the extreme form of UE, binge eating ${ }^{8}$, are comorbid with various psychiatric phenotypes, such as autism ${ }^{12}$, anxiety ${ }^{13}$, mood disorders, including depression and bipolar disorder ${ }^{14}$, as well as with avoidant, antisocial, and schizotypal personality disorders ${ }^{15}$. This raises the possibility of an underlying behavioural endophenotype that confers vulnerability to obesity, overeating, and various psychiatric phenotypes. We therefore explored the similarity of the behavioural profiles of obesity and UE with those of several possibly relevant psychiatric phenotypes.

To estimate behavioural similarity, we compared the personality profiles of each phenotype derived from a popular and comprehensive personality test, the NEO PI-R/3 ${ }^{16,17}$. We use the term behavioural similarity only for simplicity as NEO PI-R/3 summarises people's actions, as well as thoughts, feelings, and goals ${ }^{16}$. NEO PI-R/3 has 240 questions, which can be summarised 

into 30 facets, which in turn belong to five major personality domains: Neuroticism, a tendency to experience negative affect; Extraversion, a tendency to experience positive affect; Openness, a preference for novelty and intellectual curiosity; Agreeableness, a tendency for altruism; and

63 Conscientiousness, an ability to control impulses that facilitates goal-directed behaviour. A 64 phenotype's personality profile refers to the pattern of associations that this phenotype has with 65 personality traits.

66 Most profile comparison research has focused on the broad domains level where the phenotypes 67 of interest tend to be behaviourally similar. Several reviews and meta-analyses have shown the 68 tendency for alcohol consumption, smoking, obesity and different psychiatric phenotypes to have 69 a similar underlying personality profile, characterised by high Neuroticism and low 70 Conscientiousness ${ }^{3,4,18,19}$ (see also Figure 1, domain section). However, this literature ignores the 71 more fine-grained information contained in the facets that make up each domain. Two phenotypes seemingly similar based on high Neuroticism and low Conscientiousness domain scores, may, in fact, be very different in their facet-level personality profiles. This is not a mere

74 hypothetical, as obesity is associated only with specific facets within Neuroticism and 75 Conscientiousness ${ }^{\text {e.g., } 20}$. Therefore, obesity might indeed be less similar with addictions based on 76 facet-level behavioural profiles than the domain-based evidence would suggest (compare domain 77 vs facet profiles in Figure 1). 


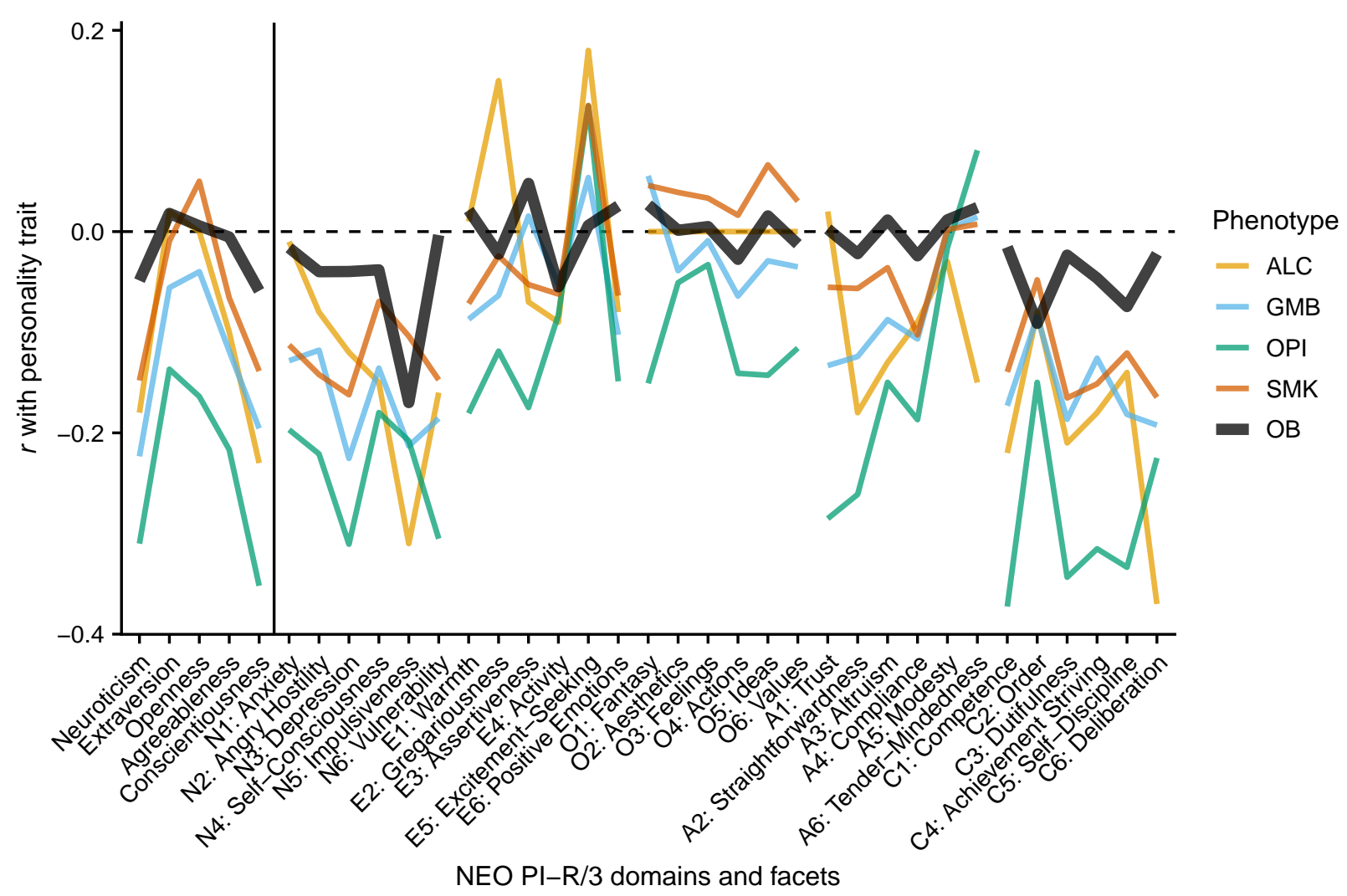

Figure 1. Personality trait profiles of obesity and selected addictions. Neuroticism has been reversed to Emotional Stability to avoid inflation of profile correlations. Solid vertical line separates domains from facets. The horizontal dashed line indicates 0 on y axis. Figure has been conceptually reproduced from Michaud et al. ${ }^{3}$. ALC = Alcohol; GMB = Gambling; OB = Obesity; OPI = Opioid abuse; $r$ = correlation; SMK = Smoking

To assess behavioural similarity between obesity, UE, addiction and psychiatric phenotypes, we “upcycled” previously published NEO PI-R/3 domain and facet profiles of each phenotype (Supplementary Table 1). The personality profiles were obtained either by comparing mean NEO PI-R/3 scores of people with a diagnosis (e.g., depression) to those of a control group, or correlating the NEO PI-R/3 facet scores with a continuous trait, such as body mass index (BMI) or a self-report questionnaire score. An example set of profiles is presented in Figure 1. We then 
formally assessed behavioural similarity of the phenotypes by correlating the personality profiles, obtaining personality correlations $\left(r_{p}\right)$ between them. For most analysis, we present the domain-based results along with the facet-based results, to compare their informativeness.

Inspecting personality correlations revealed an overall similarity in all phenotypes, but also some notable clusters among them. See Supplementary Figure 1 for domain-based personality correlations and Supplementary Figure 2 for facet-based personality correlations. The mean absolute personality correlations were stronger within the domain-based analysis (mean $r_{p}=.54$, 95\% CI $[.51, .56]$ ) than facet-based analysis (mean $r_{p}=.45$, 95\% CI [.42, .47], as confirmed by paired two-tailed $t$-test: $t(377)=9.55, p<.001, d=.49,95 \%$ CI $[.35, .64]$. This suggests that facet-level profiles demonstrate behavioural differences between phenotypes that are not evident from domain-based profiles. In other words, facets allow for greater discriminant validity among the phenotypes.

We next sought to formally assess the extent to which our target phenotypes, obesity and UE had behavioural similarities with addiction and psychiatric phenotypes. As an effect size baseline, our analysis also included education and gender to provide a "null hypothesis" for the associations. As maximum expected effect size, we considered the correlations that addiction/psychiatric phenotypes had with each other.

At the domain level, phenotypes varied in the personality correlations they had with addictions $\left(F(4,34)=11.26, p<.001, \eta_{p}^{2}=.57,95 \%\right.$ CI $\left.[.27, .68]\right)$. This is illustrated in Figures 2a and 2c, where obesity (mean $r_{p}=.73,95 \%$ CI $[.52, .94]$ ) and UE (mean $r_{p}=.67,95 \%$ CI $[.48, .86]$ ) seemed to have considerably stronger personality correlations with addictions than gender (mean $r_{p}=.42,95 \%$ CI $[.23, .61]$ ) or education (mean $r_{p}=.48,95 \%$ CI $\left.[.26, .69]\right)$. At the same time, 
there was considerable behavioural similarity between UE, obesity, and addictions, as personality correlations that UE or obesity had with addictions were of similar magnitude as those between the different addictive phenotypes (mean $r_{p}=.85,95 \%$ CI [.78, .92], Figure 2c). To statistically test the differences between the mean values in personality correlations with addictions, we repeated the ANOVA as a regression model where phenotype category predicted personality correlation strength with addictions, setting obesity or UE as a reference category (full model adjusted $R^{2}=.52,95 \%$ CI [.31,.79], $F(4,34)=11.26, p<.001$ ). We then extracted the contrasts comparing $\mathrm{OB}$ and UE with other phenotypes and corrected p-values across these contrasts with Holm correction. Education had lower personality correlations with addictions than obesity $(b=-.38,95 \%$ CI [-.57 -.18], $p=.003)$ and than UE ( $b=-.28,95 \%$ CI [-.47 -.08], $p$ $=.03)$. Similarly, gender had lower personality correlations with addictions compared to obesity $(b=-.43,95 \%$ CI [-.63 -.23], $p=.001)$ and to UE $(b=-.33$, 95\% CI [-.53 -.13], $p=.009)$. At the same time, there were no statistical differences between addiction-related personality correlations of obesity and UE ( $b=-.1,95 \%$ CI [-.3 .1], $p=.652)$, between obesity and addictions ( $b=0,95 \%$ CI [-.16 0.17], $p=.989)$, and between UE and addictions $(b=.1,95 \%$ CI [-.06 .27], $p=.652)$. All reported statistical comparisons are also reported in Supplementary Table 2. A more nuanced result emerged from facet-level analyses (Figures $2 b$ and $2 d$ ). The five groups were better separable in the personality correlations they had with addictions, as suggested by higher effect size $\left(F(4,34)=40.82, p<.001, \eta_{p}^{2}=.83,95 \%\right.$ CI $\left.[.67, .87]\right)$. Figure 2d revealed a gradient of similarities with addictions, where education (mean $r_{p}=.21,95 \%$ CI [.06, .37]) and gender (mean $r_{p}=.18$, 95\% CI $[.11, .24]$ ) were the lowest, followed by obesity (mean $r_{p}=.36$, 95\% CI $[.23, .49]$ ), which was followed by UE (mean $r_{p}=.51,95 \%$ CI $[.36, .66]$ ), and then by 
135 addictions (mean $r_{p}=.73,95 \%$ CI $[.67, .8]$ ). The gradient was confirmed when comparing 136 differences in personality correlation estimates from the regression model (adjusted $R^{2}=.81$, $13795 \%$ CI $[.73, .9], F(4,34)=40.82, p<.001$.) Regarding baseline phenotypes, gender had weaker 138 personality correlations with addictions than obesity $(b=-.22$, 95\% CI [-.36 -.09], $p=.006)$ and 139 than UE $(b=-.4,95 \%$ CI [-.54 -.27], $p<.001)$. Similarly, education had weaker personality 140 correlations with addictions compared to obesity $(b=-.22,95 \%$ CI [-.36 -.09], $p=.006)$ and 141 (UE -.4, 95\% CI [-.54 -.27], $p$ <.001). Intriguingly, UE had higher similarity to addictions than 142 obesity $(b=.18,95 \%$ CI [.04 .31], $p=.019)$. Further, addictions had even higher similarities 143 with each other than with UE $(b=.15,95 \%$ CI [.04 .26], $p=.019)$ and with obesity $(b=.33$, $14495 \%$ CI $[.22 .44], p<.001)$. 
a

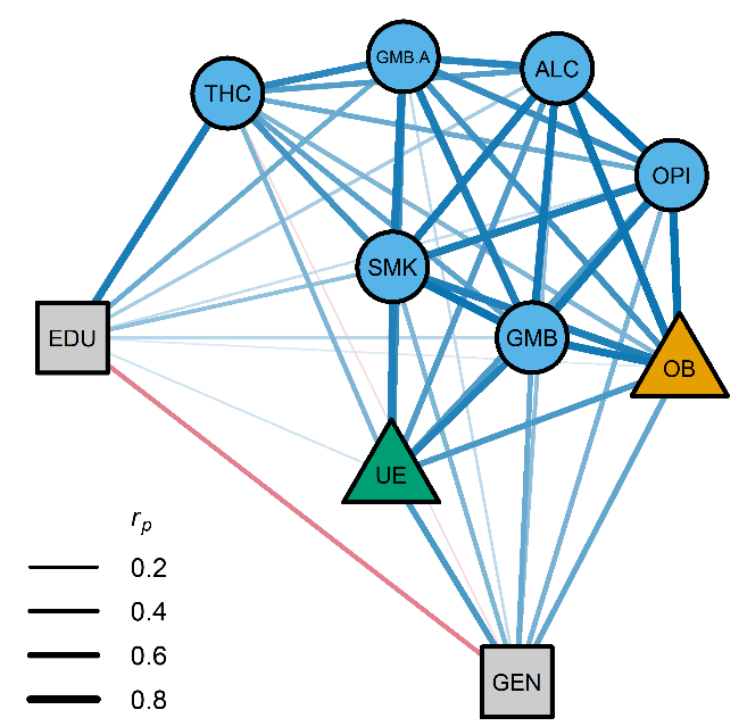

C

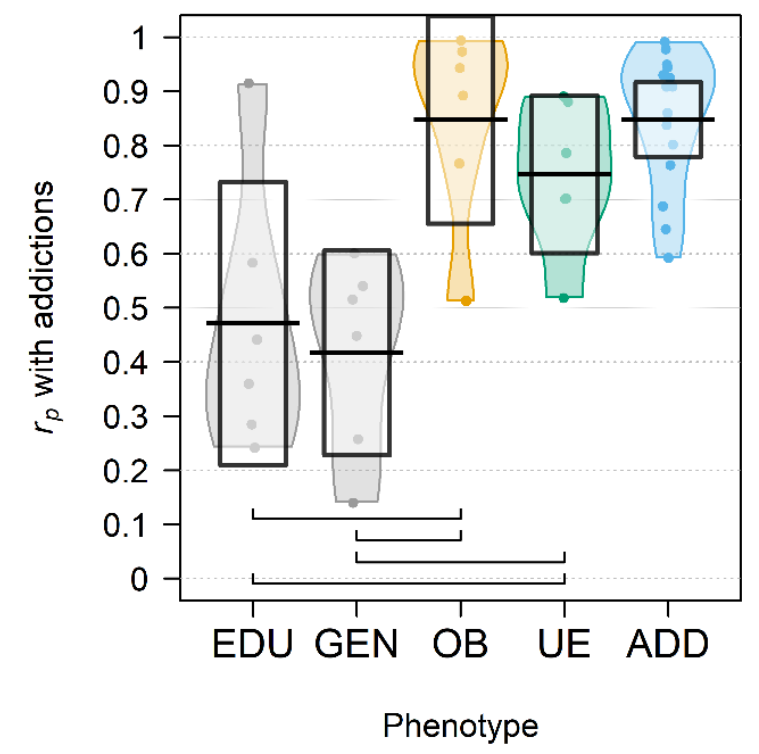

b

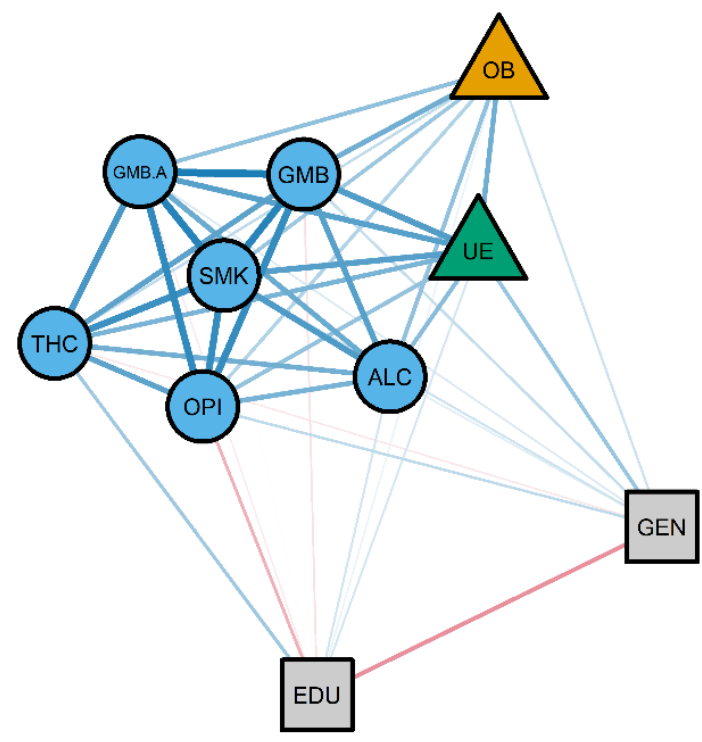

d

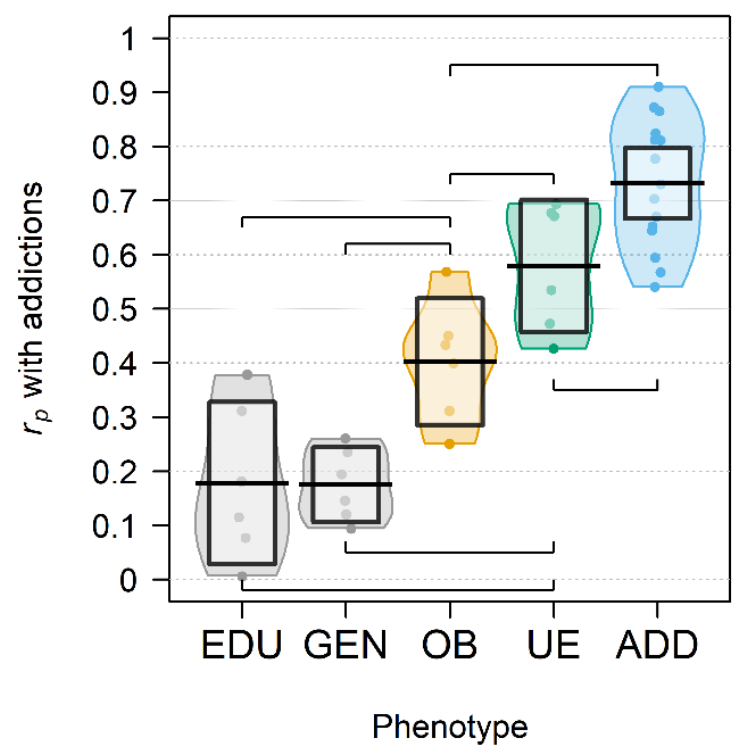

146 Figure 2. Personality correlations $\left(r_{p}\right)$ with addiction phenotypes. A and B: Spring-embedded 147 network graph based on domains (A) or facets (B), using Fruchterman-Reingold algorithm.

148 Circles mark addiction phenotypes, triangles mark targets of the behavioural similarity analysis, 149 rectangles mark baseline phenotypes. Blue edges mark positive correlations, red edges mark 150 negative correlations. C and D: Same data as in panels A and B based on domains (C) or facets (D). Correlations are in absolute values, organised by analysis targets. Points represent individual 
personality correlations, thick lines represent mean values, beans represent smoothed densities, and the rectangles represent 95\% confidence intervals. EDU, GEN, OB, UE have 6 personality correlations, ADD has 15 personality correlations. Horizontal brackets indicate significant differences in two phenotypes regarding their mean personality correlation with addiction phenotypes. Differences were detected with multiple regression, using Holm corrected $p$ value

$157<.05$. Full statistics are reported in Supplementary Table 2. ADD = Addictions; ALC = Alcohol; 158 EDU = Education; GEN = Gender; GMB = Gambling; GMB.A = Gambling with attention 159 deficit hyperactivity disorder; OB = Obesity; OPI = Opioid abuse; SMK = Smoking; $\mathrm{THC}=$ 160 Cannabis; UE = Uncontrolled Eating.

162 We repeated the analytic approach with psychiatric phenotypes other than addiction. In the 163 domain-based analysis, the five groups differed little in their behavioural similarities with 164 psychiatric condition (Figures 3a and 3c; $F(4,220)=3.30, p=.012, \eta_{p}^{2}=.06,95 \%$ CI $[0, .11]$ ).

165 Reanalysis with the regression model $\left(R^{2}=.04,95 \%\right.$ CI $\left.[0, .12], F(4,220)=3.3, p=.012\right)$

166 revealed that there were no significant differences between obesity (mean $r_{p}=.46,95 \%$ CI

$167[.35, .57])$, gender (mean $r_{p}=.49,95 \%$ CI $[.34, .65]$ ), and psychiatric conditions (mean $r_{p}=.54$, $16895 \%$ CI $[.5, .58]$ ), apart from UE (mean $r_{p}=.64,95 \%$ CI $[.51, .78]$ ) being more similar to 169 psychiatric conditions than education (mean $r_{p}=.37,95 \%$ CI $[.25, .48] ;-.31,95 \%$ CI [-.48 $170-.13], p=.007)$. The non-significant contrasts were: obesity-education: $b=-.1,95 \%$ CI $171 \quad[-.28 .08], p=.63$; obesity-gender: $b=.04,95 \%$ CI [-.14 .22], $p=.665$; obesity-UE: $b=.21$, $17295 \%$ CI [.03 .39], $p=.135$; obesity-psychiatric conditions: $b=.09$, 95\% CI [-.05 .22], $p=.63$; 
173 UE-gender: $b=-.17,95 \%$ CI [-.35 .01], $p=.319$; UE-psychiatric conditions: $b=-.12,95 \%$ CI

$174 \quad[-.26 .01], p=.319$, see also Supplementary Table 3.

175 However, facet-based analyses once again revealed more differences between obesity and UE in 176 their personality correlations with psychiatric conditions (Figures 3b and 3d, $F(4,220)=9.42, p$ $177<.001, \eta p^{2}=.15,95 \%$ CI $\left.[.06, .22]\right)$. Regression analysis of personality correlations $\left(R^{2}=.13\right.$ $17895 \%$ CI $[.07, .21], F(4,220)=9.42, p<.001)$ revealed that obesity had generally low similarity 179 with psychiatric conditions (mean $r_{p}=.24$, 95\% CI [.17, .31]). Namely, obesity had similar 180 personality correlations with psychiatric conditions like the two baseline phenotypes of gender 181 (mean $r_{p}=.32,95 \%$ CI [.23, .42]); $b=.09,95 \%$ CI [-.07 .25], $p=.794$ and education (mean $r_{p}$ $182=.27,95 \%$ CI $[.2, .34]) ; b=.03,95 \%$ CI [-.13 .19], $p=1$, and correlations among psychiatric 183 phenotypes were considerably higher than obesity's personality correlations with them $(b=.27$, $18495 \%$ CI [.15 .39], $p$ <.001). UE (mean $r_{p}=.49$, 95\% CI $[.38, .6]$ ) had higher similarity with 185 psychiatric conditions than obesity $b=.28,95 \%$ CI [.12 .44], $p=.004$ or education $b=-.25$, $18695 \%$ CI [-.41 -.09], $p=.013$, but not gender $b=-.19$, 95\% CI [-.35 -.03], $p=.085$. UE had 187 similar personality correlations with psychiatric conditions to the personality correlations 188 between psychiatric conditions (mean $r_{p}=.5$, 95\% CI $[.46, .54]$ ); $b=-.01,95 \%$ CI [-.13 .11], $p$ $189=1$ (Supplementary Table 3). This suggests that there was considerable behavioral overlap 190 between UE and many psychiatric phenotypes but obesity had generally lower similarities. 
a

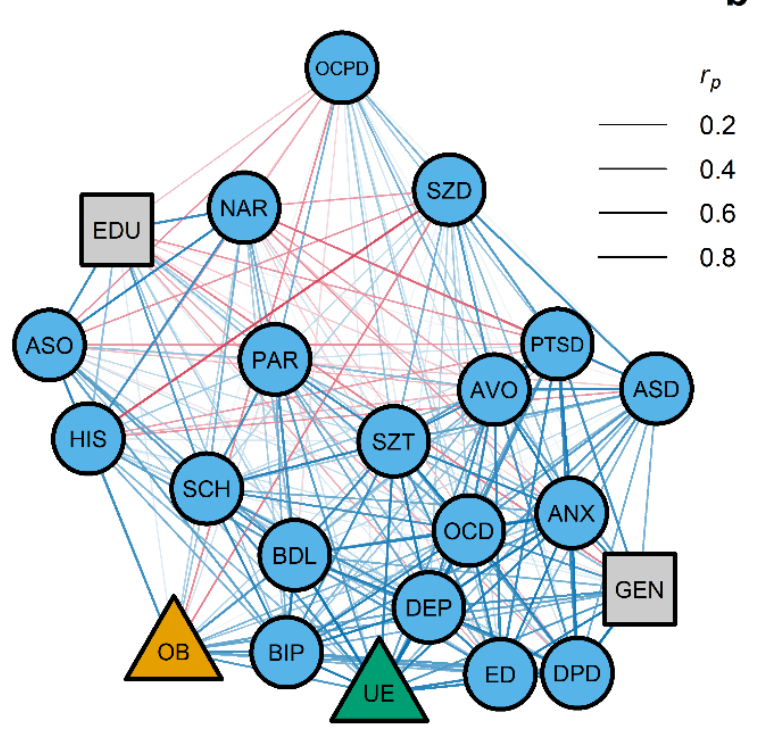

C

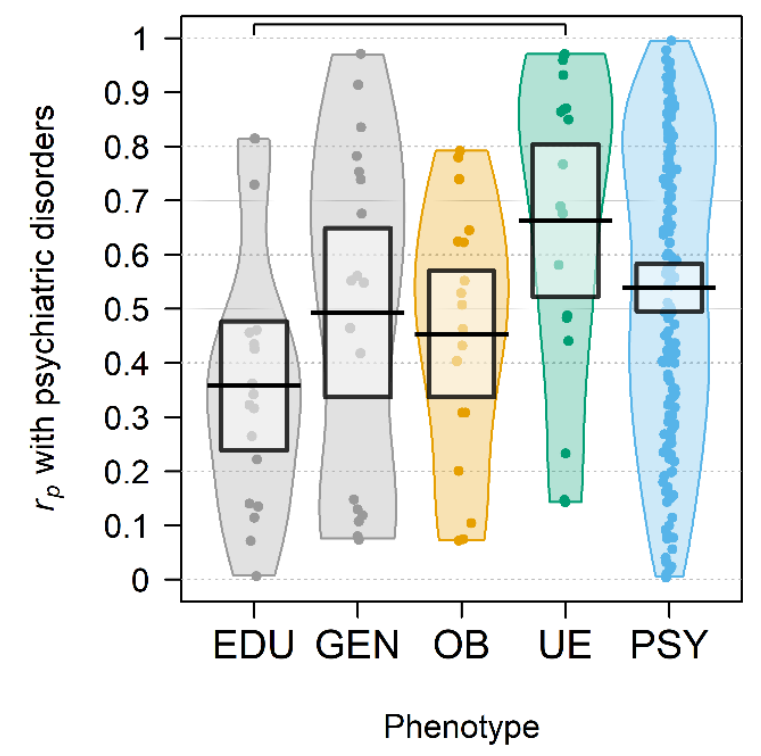

b

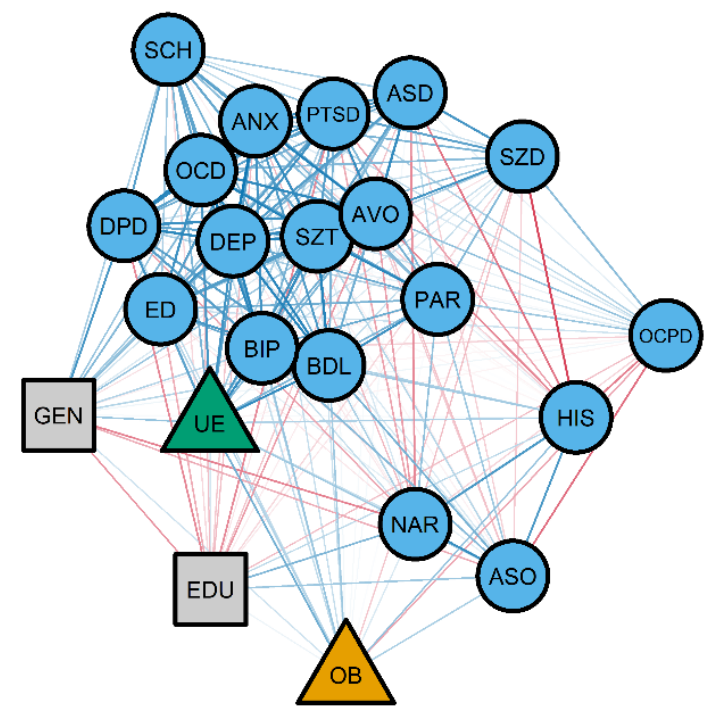

d

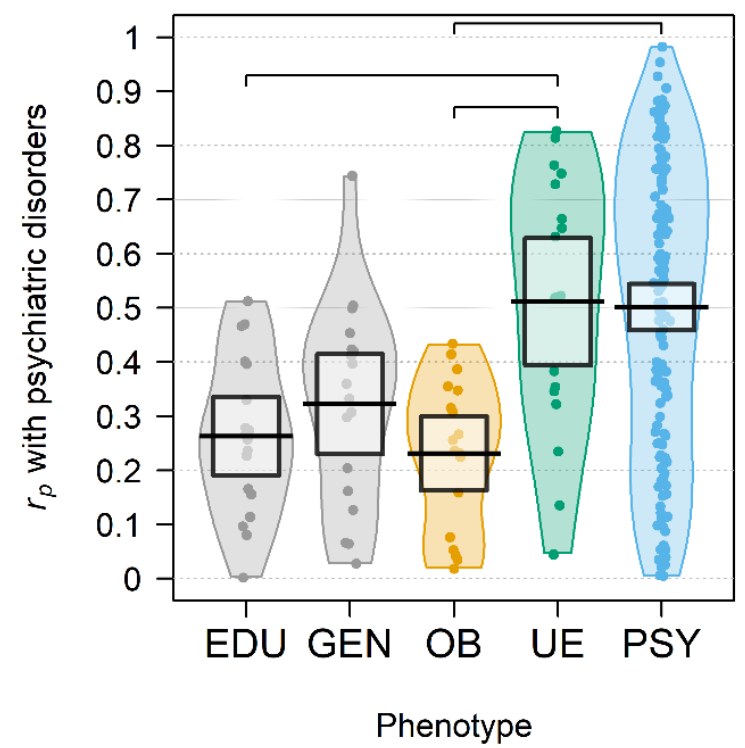

192 Figure 3. Personality correlations $\left(r_{p}\right)$ with psychiatric phenotypes. A and B: Spring-embedded

193 network graph based on domains (A) or facets (B), using Fruchterman-Reingold algorithm.

194 Circles mark psychiatric phenotypes, triangles mark targets of the behavioural similarity

195 analysis, rectangles mark baseline phenotypes. Blue edges mark positive correlations, red edges

196 mark negative correlations. C and D: Same data as in panels A and B based on domains (C) or

197 facets (D). Correlations are in absolute values, organised by analysis targets. Points represent 
individual correlations, thick lines represent mean values, beans represent smoothed densities, and the rectangles represent 95\% confidence intervals. EDU, GEN, OB, UE have 18 data points, PSY has 153 data points. Horizontal brackets indicate significant differences in two phenotypes regarding their mean personality correlation with psychiatric phenotypes. Differences were detected with multiple regression, using Holm corrected $p$ value $<.05$. Full statistics are reported in Supplementary Table 3. ANX = Anxiety disorders; ASD = Autism; ASO = Antisocial; AVO = 204 Avoidant; BDL = Borderline; BI $p=$ Bipolar; DE $p=$ Depression; DPD = Dependent ED = Nonanorexic eating disorders; EDU = Education; GEN = Gender; HIS = Histrionic; NAR = Narcissistic; OB = Obesity; OCD = Obsessive compulsive disorder; OCPD = Obsessive compulsive personality disorder; PAR = Paranoid; PSY = Psychiatric phenotypes; PTSD = Post traumatic stress disorder; SCH = Schizophrenia; SZD = Schizoid; SZT = Schizotypal; UE = 209 Uncontrolled Eating.

As exploratory analysis, we described psychiatric phenotypes whose personality correlation with obesity was observed of similar magnitude like between obesity and addictions $\left(r_{p}=.46\right.$, Figure 4c). A systematic review by Gerlach et al ${ }^{15}$ suggested that cluster $C$ personality disorders related to anxiety and fearfulness may have higher overlap with obesity than other two other personality clusters (cluster A “odd-eccentric” and cluster B “dramatic-emotional”). However, only obsessive-compulsive personality disorder from cluster $C$ had a negative association with obesity in our quantitative analysis (Figures 3b, 4f, and Supplementary Figure 2). Other correlations of similar magnitude implicated behavioural similarities between obesity and mood disorders (bipolar and borderline) and cluster B personality disorders (antisocial disorder, narcissistic disorder, histrionic disorder) (Figures 3b, 4d, and 4e, and Supplementary Figure 2). 
221 To understand which facets from the NEO PI-R/3 tended to account for the behavioral

222 similarities of addictions, obesity and UE, we plotted the facet-based behavioural profile

223 correlations. UE’s similarity with addictions (Figure 4a) was characterised by high scores on

224 Neuroticism and no associations with Openness. In contrast, obesity’s similarity with addictions

225 (Figure 4c) was mostly driven by certain specific facets: N5: Impulsiveness, C2: Order, and C5:

226 Self-discipline. Perhaps surprisingly, the E5: Excitement-Seeking facet was an outlier as it was

227 associated with addictions, but not with UE or obesity.

228 Similarly, the association between UE and psychiatric phenotypes was again driven by high

229 associations with Neuroticism and generally no associations with Openness (Figure 4b). Because

230 the behavioural similarity between obesity and psychiatric phenotypes was generally low, we

231 inspected the few psychiatric phenotypes that had a relatively higher behavioural similarity with

232 obesity (Figures 4d-f). Again, similarity in associations with specific facets, such as N5:

233 Impulsiveness, C2: Order, and C5: Self-discipline was prominent. Interestingly, obesity’s

234 association with cluster B personality disorders also highlighted that these phenotypes were

235 similar in having association with E3: Assertiveness (Figure 4d). 

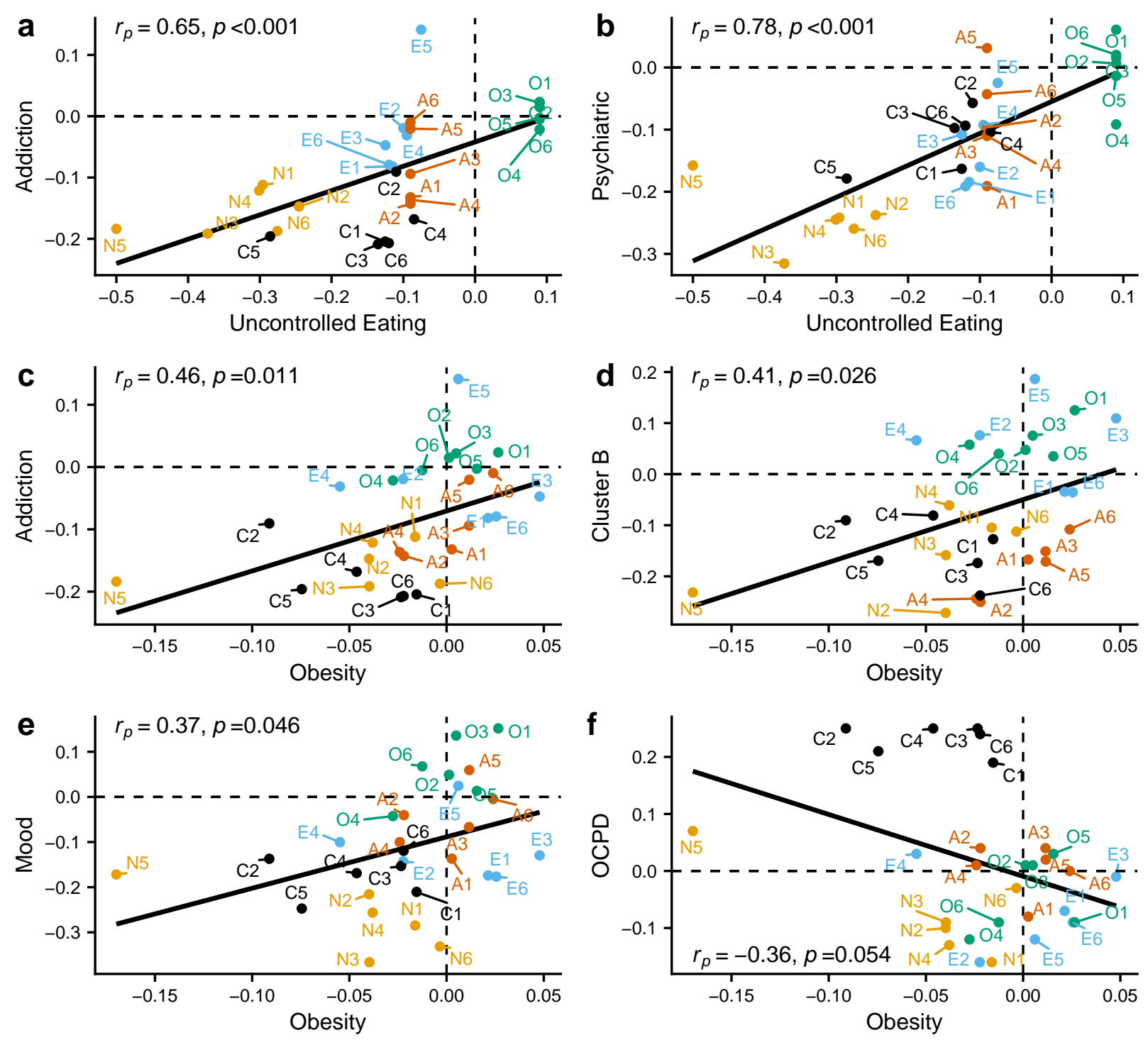

Factor $\begin{array}{lll}\text { - Neuroticism }- \text { Openness } & - \text { Conscientiousness } \\ & \text { Extraversion }- \text { Agreeableness }\end{array}$

236 Figure 4. Scatterplots of personality correlations $\left(r_{p}\right)$ between profiles of Uncontrolled Eating and 237 obesity and addiction and psychiatric phenotypes. Associations for Neuroticism were inverted to 238 avoid inflation of profile correlations as Neuroticism is keyed to the socially undesirable 239 direction, as opposed to the other four domains. $\mathrm{X}$ and $\mathrm{y}$ values represent correlations of 240 phenotypes with individual facets of NEO PI-R/3. Profiles on y axis in plots A-E have been 241 aggregated across several profiles, See Figure1, Figure2, and Supplementary Table 1 for 
242 classification. Data points represent individual personality facets, colour-coded by domain.

243 OCPD = Obsessive-compulsive personality disorder.

245 The current analysis provides a quantitative estimation of behavioural similarities between 246 obesity, UE, and addictions. This was achieved by employing the personality profile comparison 247 approach. Although the similarity was highly uniform based on five broad personality domains, 248 higher-resolution facet-based analysis revealed that behavioural overlap between obesity and 249 addiction was smaller than those of UE and addictions or among addictions themselves. Facet250 based behavioural similarity analyses further revealed that UE had considerable behavioural 251 similarity with most psychiatric phenotype tested, whereas obesity’s behavioural similarity 252 pertained to mood disorders, cluster B personality disorders and obsessive-compulsive 253 personality disorder.

254 The moderate behavioural similarity between obesity and addictions provides empirical support 255 for comparing these phenotypes in more detail ${ }^{3}$. Specifically, we also highlighted the personality 256 facets possibly characterising both obesity and addictions. The similarity was mostly driven by 257 high N5: Impulsiveness and low Conscientiousness. This is in accordance with previous 258 evidence ${ }^{18}$ and suggests that similar self-regulation therapeutic approaches can be developed for 259 both obesity and addictions ${ }^{21}$. But unlike addictions, obesity does not have a consistent 260 association with sensation-seeking ${ }^{3,18}$, here measured by the E5: Excitement-seeking facet of the 261 NEO PI-R/3, which characterises craving excitement and stimulation. Therefore, sensationseeking aspects of addictions might not translate to obesity. 
263 Intriguingly, UE had more similarity with addictions than obesity. UE may therefore be

264 considered as a useful phenotype to summarise addiction-like behaviours potentially contributing

265 to obesity. It is important to note that the UE profile was derived from questionnaires that were

266 not based on the "food addiction" concept ${ }^{22}$. Therefore, we suggest that creating and using a

267 food addiction-specific questionnaire is not crucial to understand the common substrate between

268 addictions and overeating or $\mathrm{UE}^{\text {e.g., } 23}$. In the current study, the behavioural similarity between

269 UE and addictions was driven by Neuroticism, a tendency towards negative mood states and

270 anxiety driven behaviours. Several mechanisms are possible: either traits encompassed by

271 Neuroticism could be common causes contributing to overeating and addictive behaviours, or

272 overeating and addictive behaviours contribute to a person focusing on the negative aspects of

273 these behaviours, leading to higher Neuroticism scores ${ }^{10,24}$.

274 As obesity is less addiction-centred than UE, we explored whether the behavioural profile of 275 obesity could be similar to profiles of other psychiatric phenotypes. Only a handful psychiatric 276 phenotypes seemed to have behavioural similarity with obesity with an effect size close to the 277 association between obesity and addictions. Note that the similarities were descriptive and not 278 statistically tested. These were mood disorders, cluster B personality disorders, and obsessive279 compulsive personality disorder. Their overlap with obesity was driven by associations with N5: 280 Impulsiveness and Conscientiousness. As a novel finding, similarities with cluster B personality 281 disorders was further driven by the positive association between obesity and the E3:

282 Assertiveness facet of Extraversion. While assertiveness (or dominance) has been implicated in 283 previous NEO PI-R/3 studies of obesity ${ }^{\text {e.g., } 20}$, it has not been considered in behavioural models of 284 obesity. Individuals with Cluster B personality disorders, particularly individuals with narcissism 285 are known to have an exaggerated sense of superiority ${ }^{25}$, which may explain their higher scores 
in E3: Assertiveness. At first, it is hard to imagine most people with obesity having a heightened sense of superiority, as people with obesity typically have lower self-esteem ${ }^{26}$. However, it could be speculated that low self-esteem in obesity is a response to the increased status-driven individualism ${ }^{27}$, which may be indexed by higher E3: Assertiveness. Alternatively, higher E3: Assertiveness may index increased reward sensitivity in people with obesity ${ }^{3}$. Future focused analysis will have to disentangle this association.

A caveat is that each personality correlation was based on 5 domains or 30 facets. At the same time, the “scores” for domain or facets were not single-participant data points, but average scores of at least 52 participants - at times even thousands of participants. While each correlation had only 3 or 28 degrees of freedom, it borrowed power from the studies that the average facet scores were based on. Future methodological developments are required to properly assess the role of sample sizes that correlation profiles are based on, providing more accurate standard errors and $p$ rho $=-.31,95 \%$ CI $[-.68, .18], n=18, p=.21)$.

Another caveat is that the personality profile of phenotypes may vary depending on the diagnostic instrument as well as the personality trait measure used ${ }^{28}$. This is not a major concern values for personality correlations. Until then, less emphasis should be put on their $p$ values than on their relative magnitudes. In addition, profiles based on smaller sample sizes may provide noisier estimates which lowers the personality correlations. However, there was no statistically detectable association between profiles ranked by sample size and by profiles’ average of absolute personality correlations (domains: $r h o=-.14$, 95\% CI [-.57, .35], $n=18, p=.59$; facets:

for BMI, whose behavioural profile correlates $r_{p}=.96-.99$ with other measures of adiposity ${ }^{20}$. Regarding the personality measure, current analysis was mostly limited to the NEO PI-R/3, which is intended for use in normal populations. For now, we find that these limitations are 
offset by the NEO PI-R/3 profiles' wide availability. Future research of this kind may benefit from operationalising behavioural profiles using more numerous and more specific personality characteristics, possibly operationalised as single test items (nuances) ${ }^{29}$. Indeed, as recently reviewed ${ }^{30,31}$, there is a considerable amount of reliable information present in the 240 NEO PI$\mathrm{R} / 3$ items that is lost when the single items are aggregated into the 30 facets and, especially, the five domain scores. Therefore, researchers should make their data available at the item level, work with the 30 NEO PI-R/3 facets, which "are not likely to be the ideal specification of lower level traits, but for now they are a serviceable one, with known reliability, validity, and utility.” 32 Even at the facet level, as used here, the behavioural similarity analysis can bring novel insights into similarities between phenotypes. Currently, we focused on addiction and psychiatric phenotypes as there was a priori theoretical and empirical evidence for potential overlap. In the explorations, obesity and UE can be related to any other phenotype for which a NEO PI-R/3 profile has been published. This "upcycling” approach is more cost-effective than measuring all the phenotypes and obesity in a single study. Once behavioural similarity is established, the analysis on the particular facets driving the similarity can provide insights into how the behavioural similarity emerges. These insights can inform study design when obesity and the behaviourally similar phenotypes are finally included in the same study. For instance, current results suggest that obesity, personality disorders, and E3: Assertiveness-related behaviours should be studied together in greater detail. 
addictive behaviours. However, obesity cannot only be explained by a propensity to addictive behaviours. Comparison with psychiatric phenotypes highlighted that cluster B personality disorders might bring additional insight into understanding the behavioural profile of obesity. Our study provides a general framework for quantifying the behavioural similarity across many phenotypes.

\section{Methods}

Justification for NEO PI-R/3. We chose to conduct behavioural profiling based on the 30 personality traits forming the facets of the five-factor model as operationalised in the NEO PI-R/ $3^{16,17}$. The 30 NEO PI-R/3 facets are designed to comprehensively sample aspects of behaviour related to the Five-Factor Model of personality (or the Big Five) ${ }^{33}$, and the questionnaire has been related to a wide range of phenotypes. While the questions are designed to be used in normal populations ${ }^{34}$, the NEO PI-R/3 performs surprisingly well in subpopulations with addiction or psychiatric phenotypes - theoretical factor structure can be recovered, the questionnaire has test-retest reliability, and the questionnaire is also responsive to treatment of a psychiatric condition ${ }^{35-37}$. Here we analyse the personality domain and facet profiles of phenotypes of interest based on previously published associations.

Finding papers. Studies profiling obesity, UE, addiction, and psychiatric phenotypes with the NEO PI-R/3 were searched for in Google Scholar by entering “NEO PI-R/3” together with phenotype names, such as obesity, smoking, gambling, drug use, and other phenotypes listed in Supplementary Table 1 . The goal of the search was not to be exhaustive, but to find a broad set of addiction and psychiatric phenotypes. When several papers were available on the phenotype, the effect sizes were either merged (see below), or previously calculated meta-analytic estimates were preferred over individual studies. Only papers reporting NEO PI-R/3 facet-based 
associations were included. 21 empirical papers ${ }^{20,35,38-56}$ were kept in the analysis, which analysed data from 19 different samples (Supplementary Table 1). We also included results from one meta-analysis summarising 16 different empirical studies analyzing 18 independent samples ${ }^{28}$. Altogether, the analysis is based on the summary statistics from 18,611 unique participants. Besides the phenotypes outlined in the introduction, we also included personality profiles of education and gender. As true null association between profiles cannot be expected, we provide education and gender as reference effect sizes for interpreting the effect sizes of obesity and UE. We further use personality correlations among addiction/psychiatric themselves as maximum expected correlations.

Data extraction, transformation, aggregation. Our goal was to present all associations between personality traits and phenotypes in a common metric - correlation. Correlation or another measure of effect size was readily available in fewer than half of the empirical papers ${ }^{20,38-44}$. In other papers, correlations were obtained in the following way. Most papers reported NEO PI-R/3 facet T-score means and standard errors / standard deviations (SD) for one or more study group (s) and control group. For some of the traits, multiple groups were available, for instance smokers, never smokers, and former smokers ${ }^{50}$ or underweight, normal weight, overweight, and obese ${ }^{51}$. In these cases, we focused on the phenotype group vs control group, 373 as for instance former smokers have a different personality than current and never smokers ${ }^{55}$. 374 We extracted the mean, $S D$, and sample size for study groups [psychiatric, current users, obese 375 (body mass index BMI $\geq 30 \mathrm{~kg} / \mathrm{m}^{2}$ )], and control group [never users, normal weight (BMI between 18.5 and $24.9 \mathrm{~kg} / \mathrm{m}^{2}$ ). Using control group data from the included studies was preferred, 
groups come from different countries or regions ${ }^{38}$. However, when control group data was not available, the NEO PI-R/3-R US normative sample (mean $=50, S D=10, n=1000)^{16}$ was used. US normative sample data was also used in cases where the control group consisted of participants with psychiatric disorders ${ }^{52}$. In one case, findings were available for two time points; these measurement were aggregated ${ }^{35}$. When $S D$ was not available ${ }^{35,45}$, it was calculated from standard error, or assumed to be 10 , as per the NEO PI-R/3-R manual ${ }^{16}$.

The mean difference between the study group and the control group in a personality trait was converted into a correlation in the following way. First, a summarised $t$-test was performed between the control group and the study group for each domain and facet, using the extracted means, $S D$-s and sample sizes. Unequal variances were used as per previous recommendations ${ }^{57}$. The procedure was conducted using tsum.test() from the R package BSDA ${ }^{58}$. The $t$-test was twosided with a $p$-value of .05. However, the $p$-values were not used in the further effect size conversion process. Obtained effect sizes were converted to a correlation coefficient using conversion formulas implemented in the compute.es R package, which first convert the $t$-test values into Cohen's $d$, which is then converted into a correlation, using standard formulas ${ }^{59,60}$. We tested the effect size conversion procedure using data from a paper ${ }^{20}$ that provided both trait mean and $S D$ for both groups that had either normal weight or obesity, as well as continuous trait-BMI correlations ${ }^{20}$. The trait-obesity correlations reported in that paper ${ }^{20}$ were almost identical to the trait-obesity status correlations calculated from contrasting the group having normal weight with the group having obesity $\left(r_{p}=.99\right)$.

When several papers were available, the correlations were aggregated using meta-analytic random effects aggregation. Random effects aggregation accounts for variation in study methodology between different study sites ${ }^{61}$. Before meta-analysing, correlations were 
transformed based on Fischer's $r$-to-z transformation (from Pearson- $r$ to normal distribution $z$ -

402

403

404

405

406

407

408

409

410

411

412

413

414

415

416

417 score), which is a recommended approach as $r$ is not normally distributed ${ }^{61}$. These steps were conducted by the metacor() function of the meta R package ${ }^{62,63}$. We also aggregated data for conceptually similar smaller samples, for instance phobias and anxiety disorders. There, the sample size weight was the size of the study group (see phenotype group column in Supplementary Table 1). Since many eating-related traits are highly similar ${ }^{8,9}$, we also aggregated emotional and external eating ${ }^{39}$ into UE. Some papers omitted facets with small effect size; missing facets were then replaced with domain level effect sizes. Two papers ${ }^{38,54}$ omitted domain-outcome correlations. We then used the other 26 profiles to train a model that predicted each domain-outcome correlation from the facet-outcome correlations belonging to that domain. 5-fold cross-validation within the 26 profiles revealed that mean absolute error ranged from $r=.03$ to $r=.04$ for different domains. Only self-reported profiles were used e.g., ${ }^{3}$. Data sources and meta-analytic aggregations are summarised in Supplementary Table 1.

Data analysis. Profile similarity was assessed by shape similarity, which is computed with Pearson correlations between profiles ${ }^{64}$. We focus on similarity based on profile shape, as shape is the most fundamental element for personality profile comparison and drives other similarities ${ }^{64}$. We inverted the scores of Neuroticism, to avoid inflation of profile correlations due to Neuroticism being keyed to the socially undesirable direction, while the other four domains are keyed in the socially desirable direction. For an initial presentation (Supplementary Figures S1 and S2), the resulting correlation matrix was clustered with the "warp.d2" method ${ }^{65}$. We conducted separate analysis for addiction and psychiatric phenotypes. Subsets of the main correlation matrix were visualised with a network with spring-embedded layout ${ }^{66}$ that creates clusters of more strongly related variables (Figures 1a, 1b, 2a, and 2b). 
424 We first sought to establish, if there were any differences in how addiction/psychiatric 425 phenotypes related to baseline, target, and other addiction/psychiatric phenotypes. Therefore, 426 personality correlations of addiction/psychiatric phenotypes were organised into five groups: 427 correlations with 1) education, 2) gender, 3) obesity, 4) UE, and 5) other addiction/psychiatric 428 phenotypes. Those groups were used as predictors of absolute personality correlation in a one429 way ANOVA model. Post-hoc tests were run in a linear regression model where target 430 phenotype (obesity or UE) was the reference category, whose absolute correlations with 431 addiction/psychiatric phenotypes were compared with the correlations that variables within four 432 other variable sets had with the same phenotypes (Supplementary Tables S2 and S3). $p$ values of 433 post-hoc comparisons of interest were two-sided and corrected for multiple comparison with $434 \quad$ Holm method.

435 To understand which facets of NEO PI-R/3 drive the correlations, scatterplots between the 436 profile correlations were inspected. To limit the number of scatterplots, the profiles of addiction 437 or psychiatric phenotypes were aggregated by the categories outlined in Supplementary Table 1, 438 using meta-analytic principles, but keeping the sample sizes equal (e.g. $n=100$ ), as we wanted 439 each phenotype to contribute equally to the aggregated profile.

440 All analysis was conducted in Microsoft R Open 3.5.1 ${ }^{62}$ using the August 2018 version of 441 several addon packages ${ }^{58,59,63,67-77}$. Analysis code is available as described in code availability 442 section.

444 The correlation profiles of phenotypes used in the analysis are available at https://osf.io/zfsxd/ and also as Supplementary Data and part of Supplementary Software. 
447 The analysis script used to generate results based on the correlation profiles is available at 448 https://osf.io/zfsxd/ and also as Supplementary Software

\section{References}

1. Abajobir, A. A. et al. Global, regional, and national comparative risk assessment of 84 behavioural, environmental and occupational, and metabolic risks or clusters of risks, 19902016: a systematic analysis for the Global Burden of Disease Study 2016. The Lancet 390, 1345-1422 (2017).

2. Drewnowski, A. Obesity and the food environment: dietary energy density and diet costs. Am. J. Prev. Med. 27, 154-162 (2004).

3. Michaud, A., Vainik, U., García-García, I. \& Dagher, A. Overlapping Neural Endophenotypes in Addiction and Obesity. Front. Endocrinol. 8, 1-15 (2017).

4. Vainik, U., Dagher, A., Dubé, L. \& Fellows, L. K. Neurobehavioural correlates of body mass index and eating behaviours in adults: A systematic review. Neurosci. Biobehav. Rev. 37, 279-299 (2013).

5. Blundell, J. E. \& Finlayson, G. Is susceptibility to weight gain characterized by homeostatic or hedonic risk factors for overconsumption? Physiol. Behav. 82, 21-25 (2004).

6. Volkow, N. D., Wang, G.-J., Tomasi, D. \& Baler, R. D. Obesity and addiction: neurobiological overlaps. Obes. Rev. 14, 2-18 (2013).

7. Tang, D. W., Fellows, L. K., Small, D. M. \& Dagher, A. Food and drug cues activate similar brain regions: A meta-analysis of functional MRI studies. Physiol. Behav. 106, 317-324 (2012). 
8. Vainik, U., Neseliler, S., Konstabel, K., Fellows, L. K. \& Dagher, A. Eating traits questionnaires as a continuum of a single concept. Uncontrolled eating. Appetite 90, 229_ 239 (2015).

9. Price, M., Higgs, S. \& Lee, M. Self-reported eating traits: Underlying components of food responsivity and dietary restriction are positively related to BMI. Appetite 95, 203-210 (2015).

10. Vainik, U., García-García, I. \& Dagher, A. Uncontrolled eating: a unifying heritable trait linked with obesity, overeating, personality and the brain. Eur. J. Neurosci. 0, 1-16 (2019).

11. Blundell, J. E. \& Cooling, J. Routes to obesity: Phenotypes, food choices and activity. Br. J. Nutr. 83 Suppl 1, S33-38 (2000).

12. Murphy, C. M. et al. Autism spectrum disorder in adults: diagnosis, management, and health services development. Neuropsychiatr. Dis. Treat. 12, 1669-1686 (2016).

13. Gariepy, G., Nitka, D. \& Schmitz, N. The association between obesity and anxiety disorders in the population: a systematic review and meta-analysis. Int. J. Obes. 34, 407-419 (2010).

14. McElroy, S. L. et al. Are Mood Disorders and Obesity Related? A Review for the Mental Health Professional. J. Clin. Psychiatry 65, 634-651 (2004).

15. Gerlach, G., Loeber, S. \& Herpertz, S. Personality disorders and obesity: a systematic review. Obes. Rev. 17, 691-723 (2016).

16. Costa, P. T. \& McCrae, R. R. Revised NEO Personality Inventory (NEO-PI-R) and NEO Five-Factor Inventory (NEOFFI) - professional manual. (Psychological Assessment Resources, Inc., 1992).

17. McCrae, R. R., Costa, Jr., P. T. \& Martin, T. A. The NEO-PI-3: A More Readable Revised NEO Personality Inventory. J. Pers. Assess. 84, 261-270 (2005). 
18. Emery, R. L. \& Levine, M. D. Questionnaire and behavioral task measures of impulsivity are differentially associated with body mass index: A comprehensive meta-analysis. Psychol. Bull. 143, 868-902 (2017).

19. Malouff, J. M., Thorsteinsson, E. B. \& Schutte, N. S. The Relationship Between the FiveFactor Model of Personality and Symptoms of Clinical Disorders: A Meta-Analysis. $J$. Psychopathol. Behav. Assess. 27, 101-114 (2005).

20. Sutin, A. R., Ferrucci, L., Zonderman, A. B. \& Terracciano, A. Personality and obesity across the adult life span. J. Pers. Soc. Psychol. 101, 579-592 (2011).

21. Jansen, A., Houben, K. \& Roefs, A. A Cognitive Profile of Obesity and Its Translation into New Interventions. Front. Psychol. 6, 1-9 (2015).

22. van Strien, T., Frijters, J. E. R., Bergers, G. P. A. \& Defares, P. B. The Dutch Eating Behavior Questionnaire (DEBQ) for assessment of restrained, emotional, and external eating behavior. Int. J. Eat. Disord. 5, 295-315 (1986).

23. Finlayson, G. Food addiction and obesity: unnecessary medicalization of hedonic overeating. Nat. Rev. Endocrinol. 13, 493-498 (2017).

24. Ormel, J. et al. Neuroticism and Common Mental Disorders: Meaning and Utility of a Complex Relationship. Clin. Psychol. Rev. 33, 686-697 (2013).

25. American Psychiatric Association. Diagnostic and Statistical Manual of Mental Disorders. (American Psychiatric Association, 2013). doi:10.1176/appi.books.9780890425596

26. Lillis, J., Luoma, J. B., Levin, M. E. \& Hayes, S. C. Measuring Weight Self-stigma: The Weight Self-stigma Questionnaire. Obesity 18, 971-976 (2010).

27. Lemaitre, B. Connecting the obesity and the narcissism epidemics. Med. Hypotheses 95, 1019 (2016). 
28. Samuel, D. B. \& Widiger, T. A. A meta-analytic review of the relationships between the five-factor model and DSM-IV-TR personality disorders: A facet level analysis. Clin. Psychol. Rev. 28, 1326-1342 (2008).

29. Mõttus, R., Kandler, C., Bleidorn, W., Riemann, R. \& McCrae, R. R. Personality traits below facets: The consensual validity, longitudinal stability, heritability, and utility of personality nuances. J. Pers. Soc. Psychol. 112, 474-490 (2017).

30. Mõttus, R. Towards More Rigorous Personality Trait-Outcome Research. Eur. J. Personal. 30, 292-303 (2016).

31. Mõttus, R., Bates, T. C., Condon, D. M., Mroczek, D., \& Revelle, W. Leveraging a more nuanced view of personality: Narrow characteristics predict and explain variance in life outcomes. Preprint at https://doi.org/10.31234/osf.io/4q9gv (In revision).

32. McCrae, R. R. The Place of the FFM in Personality Psychology. Psychol. Inq. 21, 57-64 (2010).

33. John, O. \& Srivastava, S. The Big Five trait taxonomy: History, measurement, and theoretical perspectives. in Handbook of personality: Theory and research 102-138 (Guilford Press, 1999).

34. Haigler, E. D. \& Widiger, T. A. Experimental Manipulation of NEO-PI-R Items. J. Pers. Assess. 77, 339-358 (2001).

35. Carter, J. A. et al. Short-term stability of NEO-PI-R personality trait scores in opioiddependent outpatients. Psychol. Addict. Behav. 15, 255-260 (2001).

36. Costa, P. T., Bagby, R. M., Herbst, J. H. \& McCrae, R. R. Personality self-reports are concurrently reliable and valid during acute depressive episodes. J. Affect. Disord. 89, 45-55 (2005). 
37. Kentros, M. et al. Stability of personality traits in schizophrenia and schizoaffective disorder: a pilot project. J. Nerv. Ment. Dis. 185, 549-555 (1997).

38. Costa, P. J., Terracciano, A. \& McCrae, R. R. Gender differences in personality traits across cultures: Robust and surprising findings. J. Pers. Soc. Psychol. 81, 322-331 (2001).

39. Elfhag, K. \& Morey, L. C. Personality traits and eating behavior in the obese: Poor selfcontrol in emotional and external eating but personality assets in restrained eating. Eat. Behav. 9, 285-293 (2008).

40. Mõttus, R., Realo, A., Vainik, U., Allik, J. \& Esko, T. Educational Attainment and Personality Are Genetically Intertwined. Psychol. Sci. 28, 1631-1639 (2017).

41. Ruiz, M. A., Pincus, A. L. \& Dickinson, K. A. NEO PI-R Predictors of Alcohol Use and Alcohol-Related Problems. J. Pers. Assess. 81, 226-236 (2003).

42. Sutin, A. R. et al. The association between personality traits and body mass index varies with nativity among individuals of Mexican origin. Appetite (2015). doi:10.1016/j.appet.2015.02.036

43. Vainik, U., Mõttus, R., Allik, J., Esko, T. \& Realo, A. Are Trait-Outcome Associations Caused by Scales or Particular Items? Example Analysis of Personality Facets and BMI. Eur. J. Personal. 29, 688-634 (2015).

44. Wakabayashi, A., Baron-Cohen, S. \& Wheelwright, S. Are autistic traits an independent personality dimension? A study of the Autism-Spectrum Quotient (AQ) and the NEO-PI-R. Personal. Individ. Differ. 41, 873-883 (2006).

45. Bienvenu, O. J. et al. Anxiety and depressive disorders and the five-factor model of personality: A higher- and lower-order personality trait investigation in a community sample. Depress. Anxiety 20, 92-97 (2004). 
46. Davtian, M., Reid, R. C. \& Fong, T. W. Investigating facets of personality in adult pathological gamblers with ADHD. Neuropsychiatry 2, 163-174 (2012).

47. Rector, N. A., Bagby, R. M., Huta, V. \& Ayearst, L. E. Examination of the trait facets of the five-factor model in discriminating specific mood and anxiety disorders. Psychiatry Res. 199, 131-139 (2012).

48. Quirk, S. W., Christiansen, N. D., Wagner, S. H. \& McNulty, J. L. On the usefulness of measures of normal personality for clinical assessment: evidence of the incremental validity of the Revised NEO Personality Inventory. Psychol. Assess. 15, 311-325 (2003).

49. Bagby, R. M. et al. Relationship between the five-factor model of personality and unipolar, bipolar and schizophrenic patients. Psychiatry Res. 70, 83-94 (1997).

50. Terracciano, A., Löckenhoff, C. E., Crum, R. M., Bienvenu, O. J. \& Costa, P. T. Five-Factor Model personality profiles of drug users. BMC Psychiatry 8, 22 (2008).

51. Terracciano, A. et al. Facets of personality linked to underweight and overweight. Psychosom. Med. 71, 682-689 (2009).

52. Morey, L. C. et al. The representation of borderline, avoidant, obsessive-compulsive, and schizotypal personality disorders by the five-factor model. J. Personal. Disord. 16, 215-234 (2002).

53. Rector, N. A., Hood, K., Richter, M. A. \& Michael Bagby, R. Obsessive-compulsive disorder and the five-factor model of personality: distinction and overlap with major depressive disorder. Behav. Res. Ther. 40, 1205-1219 (2002).

54. Levallius, J., Clinton, D., Bäckström, M. \& Norring, C. Who do you think you are? Personality in eating disordered patients. J. Eat. Disord. 3, (2015). 
55. Terracciano, A. \& Costa, P. T. Smoking and the Five-Factor Model of Personality. Addict. Abingdon Engl. 99, 472-481 (2004).

56. Bagby, R. M. et al. Pathological gambling and the five-factor model of personality. Personal. Individ. Differ. 43, 873-880 (2007).

57. Ruxton, G. D. The unequal variance t-test is an underused alternative to Student's t-test and the Mann-Whitney U test. Behav. Ecol. 17, 688-690 (2006).

58. Arnholt, A. T. BSDA: Basic Statistics and Data Analysis. (2012).

59. Re, A. D. compute.es: Compute Effect Sizes. (2014).

60. Borenstein, M. Effect sizes for continuous data. in The handbook of research synthesis and meta-analysis, 2nd ed (eds. Cooper, H., Hedges, L. V. \& Valentine, J. C.) 221-235 (Russell Sage Foundation, 2009).

61. Shadish, W. R. \& Haddock, C. K. Combining estimates of effect size. in The handbook of research synthesis and meta-analysis, 2nd ed (eds. Cooper, H., Hedges, L. V. \& Valentine, J. C.) 257-277 (Russell Sage Foundation, 2009).

62. R Core Team. R: A language and environment for statistical computing. R Foundation for Statistical Computing, Vienna, Austria. (2013).

63. Schwarzer, G. meta: General Package for Meta-Analysis. (2017).

64. Furr, R. M. The Double-Entry Intraclass Correlation as an Index of Profile Similarity: Meaning, Limitations, and Alternatives. J. Pers. Assess. 92, 1-15 (2010).

65. Murtagh, F. \& Legendre, P. Ward’s Hierarchical Agglomerative Clustering Method: Which Algorithms Implement Ward’s Criterion? J. Classif. 31, 274-295 (2014).

66. Fruchterman, T. M. J. \& Reingold, E. M. Graph drawing by force-directed placement. Softw. Pract. Exp. 21, 1129-1164 (1991). 
605 67. Epskamp, S. et al. qgraph: Network visualizations of relationships in psychometric data. $J$. 606 Stat. Softw. 48, 1-18 (2012).

607 68. Phillips, N. yarrr: A Companion to the e-Book 'YaRrr!: The Pirate's Guide to R'. (2017).

608 69. Revelle, W. psych: Procedures for Psychological, Psychometric, and Personality Research. $609 \quad$ (2014).

610 70. Wei, T. \& Simko, V. corrplot: Visualization of a Correlation Matrix. (2016).

611 71. Wickham, H. \& RStudio. tidyverse: Easily Install and Load the 'Tidyverse'. (2017).

612 72. Wilke, C. O. \& Wickham, H. cowplot: Streamlined Plot Theme and Plot Annotations for 613 'ggplot2'. (2016).

614 73. Partners, P. Imvar: Linear Regression with Non-Constant Variances. (2018).

615 74. Gromer, D. apa: Format Outputs of Statistical Tests According to APA Guidelines. (2019).

616 75. Stanley, D. apaTables: Create American Psychological Association (APA) Style Tables. $617 \quad$ (2018).

618 76. Torchiano, M. effsize: Efficient Effect Size Computation. (2018).

619 77. Slowikowski, K. et al. ggrepel: Automatically Position Non-Overlapping Text Labels with 620 'ggplot2’. (2018).

621 
622 Acknowledgements

623 We would like to thank Jüri Allik, Kenn Konstabel, Margit Kõiv-Vainik, and Taavi Tillmann for

624 their helpful comments on the manuscript.

625 Uku Vainik was supported by Personal Post-doctoral Research Funding project PUTJD654 and

626 by Fonds de recherche du Québec - Santé (FRQS) foreign post-doctoral training award.

627 Andréanne Michaud was supported by Canadian Institutes of Health Research (CIHR). This

628 work was supported by a CIHR Foundation Scheme award to Alain Dagher. The funders had no

629 role in the conceptualization, design, data collection, analysis, decision to publish, or preparation

630 of the manuscript.

$631 \quad$ Competing interests

632 The authors declare no competing interests

633 Author contributions.

634 All authors read and contributed significantly to the manuscript and approved the submitted

635 version.

636 U.V: collected data, analysed data, wrote the paper.

637 B.M: contributed to data analysis

638 Y.Z.: contributed to data analysis methods

639 A.M.: contributed to interpretation

640 R.M.: contributed to data analysis methods and interpretation 
641 A.D.: contributed to data analysis methods and interpretation

642

$643 \quad$ Figure legends

644 Figure 1. Personality trait profiles of obesity and selected addictions. Neuroticism has been

645 reversed to Emotional Stability to avoid inflation of profile correlations. Solid vertical line

646 separates domains from facets. Figure has been conceptually reproduced from Michaud et al. 3.

647 ALC = Alcohol; GMB = Gambling; OB = Obesity; OPI = Opioid abuse; $r=$ correlation; SMK =

$648 \quad$ Smoking

649 Figure 2. Personality correlations $\left(r_{p}\right)$ with addiction phenotypes. A and B: Spring-embedded

650 network graph based on domains (A) or facets (B), using Fruchterman-Reingold algorithm.

651 Circles mark addiction phenotypes, triangles mark targets of the behavioural similarity analysis,

652 rectangles mark baseline phenotypes. Blue edges mark positive correlations, red edges mark

653 negative correlations. C and D: Same data as in panels A and B based on domains (C) or facets

654 (D). Correlations are in absolute values, organised by analysis targets. Points represent individual

655 personality correlations, thick lines represent mean values, beans represent smoothed densities,

656 and the rectangles represent 95\% confidence intervals. EDU, GEN, OB, UE have 6 personality

657 correlations, ADD has 15 personality correlations. Horizontal brackets indicate significant

658 differences in two phenotypes regarding their mean personality correlation with addiction

659 phenotypes. Differences were detected with multiple regression, using Holm corrected $p$ value

$660<.05$. Full statistics are reported in Supplementary Table 2. ADD = Addictions; ALC = Alcohol;

661 EDU = Education; GEN = Gender; GMB = Gambling; GMB.A = Gambling with attention

662 deficit hyperactivity disorder; OB = Obesity; OPI = Opioid abuse; SMK = Smoking; THC =

663 Cannabis; UE = Uncontrolled Eating. 
664 Figure 3. Personality correlations $\left(r_{p}\right)$ with psychiatric phenotypes. A and B: Spring-embedded 665 network graph based on domains (A) or facets (B), using Fruchterman-Reingold algorithm.

666 Circles mark psychiatric phenotypes, triangles mark targets of the behavioural similarity 667 analysis, rectangles mark baseline phenotypes. Blue edges mark positive correlations, red edges 668 mark negative correlations. C and D: Same data as in panels A and B based on domains (C) or 669 facets (D). Correlations are in absolute values, organised by analysis targets. Points represent 670 individual correlations, thick lines represent mean values, beans represent smoothed densities, 671 and the rectangles represent 95\% confidence intervals. EDU, GEN, OB, UE have 18 data points, 672 PSY has 153 data points. Horizontal brackets indicate significant differences in two phenotypes 673 regarding their mean personality correlation with psychiatric phenotypes. Differences were 674 detected with multiple regression, using Holm corrected $p$ value $<.05$. Full statistics are reported 675 in Supplementary Table 3. ANX = Anxiety disorders; ASD = Autism; ASO = Antisocial; AVO = 676 Avoidant; BDL = Borderline; BIP = Bipolar; DEP = Depression; DPD = Dependent; ED = Non677 anorexic eating disorders; EDU = Education; GEN = Gender; HIS = Histrionic; NAR = 678 Narcissistic; OB = Obesity OCD = Obsessive compulsive disorder; OCPD = Obsessive 679 compulsive personality disorder; PAR = Paranoid; PSY = Psychiatric phenotypes; PTSD = Post 680 traumatic stress disorder; SCH = Schizophrenia; SZD = Schizoid; SZT = Schizotypal; UE = $681 \quad$ Uncontrolled Eating.

682 Figure 4. Scatterplots of personality correlations $\left(r_{p}\right)$ between profiles of Uncontrolled Eating 683 and obesity and addiction and psychiatric phenotypes. Associations for Neuroticism were 684 inverted to avoid inflation of profile correlations as Neuroticism is keyed to the socially 685 undesirable direction, as opposed to the other four domains. X and y values represent 686 correlations of phenotypes with individual facets of NEO PI-R/3. Profiles on y axis in plots A-E 
687 have been aggregated across several profiles, See Figure1, Figure2, and Supplementary Table 1 688 for classification. Data points represent individual personality facets, colour-coded by domain. 689 OCPD = Obsessive-compulsive personality disorder. 